\title{
PESO AO NASCER, INDICE PONDERAL DE RÖHRER E CRESCIMENTO PÓS-NATAL
}

\author{
Arnaldo Augusto Franco de Siqueira* \\ Pedro Augusto Marcondes de Almeida * \\ Januário de Andrade* \\ Ana Cristina d'Andretta Tanaka *
}

RSPUB9/513

Siqueira, A. A. F. de et al. Peso ao nascer, indice ponderal de Röhrer e crescimento pós-natal. Rev. Saúde públ., S. Paulo, 14:333-42, 1980.

RESUMO: Foi estudada a relação entre peso ao nascer, indice ponderal de Röhrer e o crescimento de crianças no primeiro ano de vida, em 2 grupos de crianças. Verificou-se que crianças que nasceram com indice ponderal baixo (2,50 e menos) e peso igual ou inferior a $3.000 \mathrm{~g}$. apresentaram um retardo no crescimento, medido pelo peso, pois aos 12 meses pesaram cerca de $1.500 \mathrm{~g}$. a menos que as que nasceram com indice ponderal normal $(2,51$ e mais). Crianfas que nasceram com indice ponderal normal, qualquer que tivesse sido o peso ao nascer, no final do primeiro ano de vida apresentavam, nos 2 grupos, o mesmo peso. Concluiu-se que crianças que nasceram com indice ponderal igual ou menor que 2,50 e, ao mesmo tempo, com peso igual ou inferior a $3.000 \mathrm{~g}$. apresentaram um retardo do crescimento, medido pelo peso. A grande vantagem da técnica utilizada foi o fato de que não houve necessidade da determinação da idade gestacional.

UnItermos: Peso ao nascer. Crescimento. Lactentes de baixo peso ao nascer.

\section{INTRODUCAO}

E notória a importância do peșo do recém-nascido ao nascer, uma vez que diversos autores mostraram haver relação entre peso ao nascer e mortalidade, como Chase ${ }^{3}$, Puffer e Serrano ${ }^{17}$, Yerushalmy 22 e Niswander e Jackson ${ }^{16}$, entre outros. Chase 3 chega a defini-lo como a variável de maior importância na mortalidade neonatal e sugere que a principal razão por que a mortalidade neonatal na Holanda seja menor que a dos Estados Unidos reside na maior proporção de recém-nascidos de baixo peso que ocorre neste último país.
Siqueira 18 mostrou que, em São Paulo, mais de $60 \%$ dos recém-nascidos que morrem antes de 28 dias de idade nasceram com peso igual ou inferior a $2.500 \mathrm{~g}$.

Monteiro 15 mostrou que a proporção de recém-nascidos de baixo peso (igual ou menor que $2.500 \mathrm{~g}$ ), entre nascidos vivos, em São Paulo, é menor que $10 \%$.

Em outras palavras, os recém-nascidos de baixo peso, que correspondem a menos de $10 \%$ dos nascimentos vivos, respondem por $60 \%$ dos óbitos do período neonatal.

* Do Departamento de Saúde Materno-Infantil da Faculdade de Saúde Pública da USP - Av. Dr. Arnaldo, 715 - 01255 - São Paulo, SP - Brasil. 
SIQUEIRA, A. A. F. de et al. Peso ao nascer, indice nonderal de Röhrer e crescimento pós-natal. Rev. Saúde públ., S. Paulo, 14:333-42, 1980.

Para Chase ${ }^{3}$, o risco de morrer no periodo neonatal é cerca de 20 vezes maior para recém-nascidos de baixo peso do que para os demais nascidos vivos.

Puffer e Serrano ${ }^{17}$, mais recentemente verificaram, na Califórnia, que esse risco era, para recém-nascidos de baixo peso, 40 vezes maior do que para crianças de 2.501 $g$ e mais ao nascer.

$\mathrm{Na}$ década de 1960 surgiram vários trabalhos que mostraram haver diferenças no ritmo de crescimento fetal, dos quais o mais conhecido, senão o primeiro, foi o de Lubchenco ${ }^{9}$, que construiu uma curva de crescimento intra-uterino. Para essa autora os recém-nascidos cujos pesos se situem abaixo do percentil 10 , em cada idade gestacional, devem ser considerados pequenos para a idade gestacional.

Após os trabalhos de Lubchenco ${ }^{9}$, Usher e McLean ${ }^{20}$, Lindell ${ }^{8}$, Tanner e Thomson 19 e Gruenwald ${ }^{\circ}$, entre outros, os recém-nascidos pequenos para a idade gestacional foram sendo estudados e a maioria dos autores que se preocuparam com essas crianças verificaram, como Beck ${ }^{1}$, que o ritmo insatisfatório de crescimento se mantinha e essas crianças apresentavam sempre uma diferença significativa para menos no seu tamanho, quando comparadas com um grupo controle constituído de crianças nascidas com peso maior que $3.000 \mathrm{~g}$.

Beck ${ }^{1}$ mostrou também que crianças de bajxo peso, com crescimento intra-uterino normal, ou seja, prematuros verdadeiros, apresentam um crescimento pós-natal de tal ordem que, ao fim de um ano, a diferença diminuiu consideravelmente e que aos 10 anos não há mais diferença de tamanho entre prematuros verdadeiros e controles.

O prejuizo no crescimento pós-natal é, para vários autores, uma das conseqüências de uma "performance" metabólica inadequada, como foi visto por Chow ${ }^{4}$, Hsueh ? $e$ Winick 21 entre outros.

Para Winick 21, Dobbing 5 e Hsueh ', 0 problema đo crescimento insatisfatório (intra-uterino ou pós-natal) costuma vir acompanhado de um retardo no desenvolvimento neuro-psicomotor, muito bem estudado por Birch e Gussow 2 quanto às suas conseqüências no aprendizado escolar.

Recentemente, Miller e Hassanein 12,13,14 publicaram uma série de trabalhos a respeito do indice ponderal de Röhrer em recém-nascidos, que nada mais é que uma sofisticação da relação peso/altura.

Para Miller e Hassanein ${ }^{12,13,14}$ o diagnóstico do crescimento fetal apenas baseado no peso oferece erros pela nāo consideração do comprimento do recém-nascido. Para esses autores um recém-nascido de $2.600 \mathrm{~g}$ e $46 \mathrm{~cm}$ ao nascer é diferente de outro com o mesmo peso e $51 \mathrm{~cm}$, por exemplo.

Faltou a esses autores um acompanhamento pós-natal para saber se o indice ponderal tem alguma relação com o ritmo de crescimento após o nascimento, no primeiro ano de vida.

O propósito deste trabalho é saber se o conhecimento do indice ponderal de Röhrer ao nascer traz algum subsídio para o prognóstico do crescimento pós-natal.

\section{MATERIAL E METODOS}

Foram utilizadas duas amostras de crianças. A primeira foi constituida de crianças matriculadas no Centro de Saúde Geraldo Paula Souza, da Faculdade de Saúde Pública da USP, de 119 casos, e que formaram - Grupo Paula Souza; e a segunda, de crianças atendidas num consultório particular, em São Paulo, num total de 60 crianças, correspondendo ao Grupo Consultório.

O estudo foi retrospectivo e, no Grupo Paula Souza foram incluidas todas as crianças matriculadas de 1974 a 1978 no Centro de Saúde Geraldo de Paula Souza que tivessem peso e comprimento ao nascer anotados na ficha e que tivessem sido atendidas regularmente até 12 meses de idade, com um mínimo de 5 consultas, sendo pelo menos uma no $10^{\circ}$ mês de vida em 
diante. No consultório foram seguidos os mesmos critérios e utilizados casos de 1976 a 1978 .

Foram descartados cerca de 750 casos que não preencheram a algum desses requisitos. Desses, a maioria não dispunha de informações sobre comprimento ao nascer.

A seguir foram calculados os valores médios de peso por idade nos dois grupos.

Para cada criança foi calculado o indice ponderal (IP) de Röhrer ao nascer.

O IP é dado pela fórmula:

$$
\mathrm{IP}=\frac{\text { Peso (em gramas) } \times 100}{(\text { comprimento } \mathrm{em} \mathrm{cm})^{2}}
$$

A seguir, as crianças em cada grupo foram divididas em subgrupos: índice ponderal baixo (2,50 e menos) e indice ponderal normal (2,51 e mais).

$O$ peso ao nascer foi considerado adequado (3.001 g. e mais) ou inadequado (3.000 g. e menos).

\section{RESULTADOS E DISCUSSAO}

Os resultados constituem as Tabelas 1 a $5 \mathrm{e}$ as Figs. 1 e 2.

T A B E L A J

fndice ponderal e peso do recém-nascido ao nascer, observados nos Gr pos Paula Souze e Consultório, São Paulo, nos períodos de 1974/78 e 1976/78, respectivamente.

\begin{tabular}{|c|c|c|c|c|c|c|c|c|c|}
\hline \multirow{3}{*}{ Indice } & \multirow[t]{3}{*}{ Grupos: } & \multicolumn{2}{|c|}{ Grupo } & \multicolumn{2}{|c|}{ Paula Souza } & \multicolumn{2}{|c|}{ Grupo } & \multicolumn{2}{|c|}{ Consultório } \\
\hline & & \multicolumn{2}{|c|}{$\begin{array}{c}\text { Peso } \\
\text { adequado }\end{array}$} & \multicolumn{2}{|c|}{$\begin{array}{c}\text { Peso } \\
\text { inadequado }\end{array}$} & \multicolumn{2}{|c|}{$\begin{array}{c}\text { Peso } \\
\text { adequado }\end{array}$} & \multicolumn{2}{|c|}{$\begin{array}{c}\text { Peso } \\
\text { inatequado }\end{array}$} \\
\hline & & No & $\%$ & Ne & $\%$ & No & $\%$ & Ne & $\%$ \\
\hline 2,50 & e menos & 14 & $\mathbf{5 3 , 8}$ & 12 & 46,2 & 6 & 46,2 & 7 & 53,8 \\
\hline 2,51 & e mais & 66 & 71,0 & 27 & 29,0 & 33 & 70,2 & 14 & 29,8 \\
\hline
\end{tabular}

A Tabela 1 mostra, nos dois grupos, que cerca de metade dos recém-nascidos de indice ponderal 2,50 e menos tem peso inadequado. A Tabela mostra ainda que quase $30 \%$ dos recém-nascidos com IP 2,51 e mais tem peso inadequado, também nos dois grupos. Existe, assim, uma associação entre indice ponderal baixo e peso insuficiente do recém-nascido.

Com a mesma subdivisão dos grupos, foi estudado o peso até o fim do primeiro ano de vida.

As Tabelas 2 e 3 e Fig. 1 mostram resultados muito semelhantes para os grupos Paula Souza e Consultório, respectivamente.
É importante constatar que a diferença para menos que existia no peso do recém-nascido, para os dois subgrupos "peso inadequado", aumentou durante o primeiro ano de vida e atingiu quase $1.000 \mathrm{~g}$. no Grupo Paula Souza e 1.900 g. no Grupo Consultório.

Em outras palavras, os recém-nascidos de peso inadequado não apenas não recuperaram peso em relação aos de peso adequado mas a diferença de pesos entre os dois subgrupos aumenta tanto no Grupo Paula Souza como no Grupo Consultório.

Além disso os pesos, mês a mês, dos subgrupos "peso ao nascer adequado" são muito bons, se comparados aos padrões tradicionais ${ }^{0}$. E como se houvesse um 


\begin{tabular}{|c|c|c|c|c|c|c|c|c|c|}
\hline & 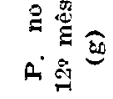 & 疍 & 密 & 善 & & 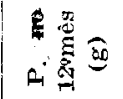 & 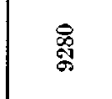 & 当 & 产 \\
\hline & 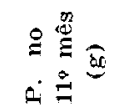 & 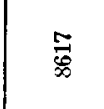 & 黄 & 高 & & 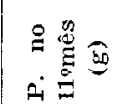 & \&్హ్ & 畄 & 旁 \\
\hline & 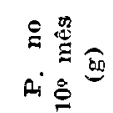 & 㞵 & $\stackrel{\infty}{\Xi}$ & 善 & & 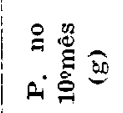 & 第 & ? & $\frac{N}{\partial}$ \\
\hline 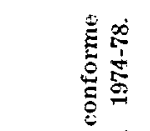 & 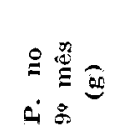 & 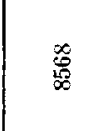 & 产 & 添 & 雚 & 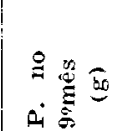 & 咅 & 管 & 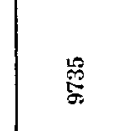 \\
\hline 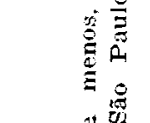 & 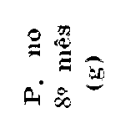 & 藃 & 管 & $\overline{\bar{x}}$ & 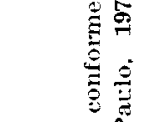 & 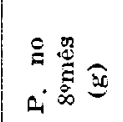 & 孚 & $\widehat{\hat{E}}$ & 罱 \\
\hline 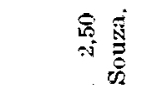 & $\cong \overline{\underline{E}}$ & 蛋 & 㤐 & $\stackrel{\infty}{\frac{\partial}{x}}$ & 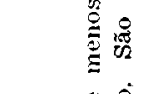 & 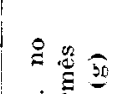 & 管 & 番 & 余 \\
\hline 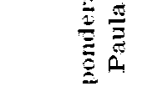 & 号笙 & $\frac{\pi}{2}$ & & & 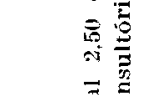 & & & & \\
\hline 畄 & 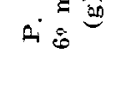 & & 莈 & $\bar{\Sigma}$ & 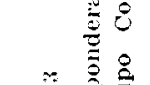 & Q & 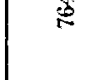 & 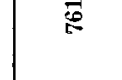 & 管 \\
\hline $4 \frac{8}{8}$ & 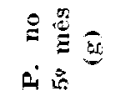 & 莫 & 纙 & 意 & 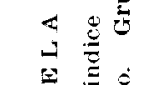 & $\stackrel{0}{=}$ & 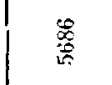 & 蕆 & 曾 \\
\hline 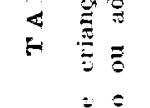 & 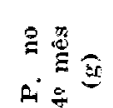 & 量 & 辤 & 绻 & 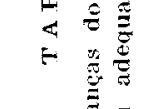 & 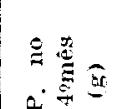 & 渃 & 惹 & 䉛 \\
\hline 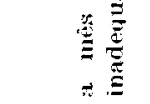 & 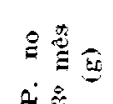 & 寽 & 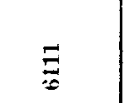 & 鍂 & 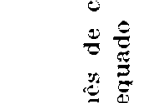 & $\cong$ & 罹 & 咅 & 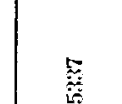 \\
\hline & 啳 & $\overrightarrow{\mathrm{D}}$ & 串 & 等 & 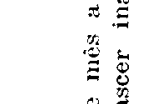 & 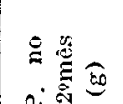 & 第 & 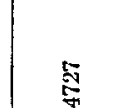 & 喓 \\
\hline & 践 & 跑 & 浔 & 畄 & 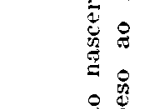 & $\cong$ & 䎏 & 齐 & 瑶 \\
\hline & 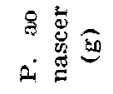 & 器 & 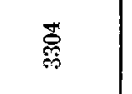 & $\vec{\nabla}$ & & 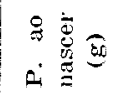 & 递 & 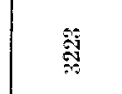 & 憘 \\
\hline & 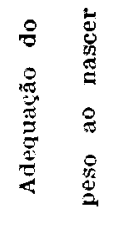 & 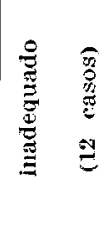 & 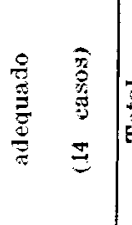 & 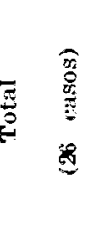 & & 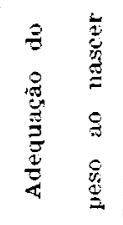 & 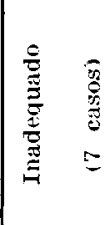 & 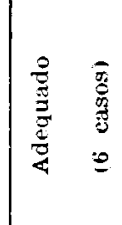 & 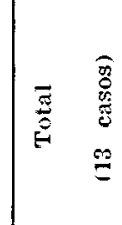 \\
\hline
\end{tabular}




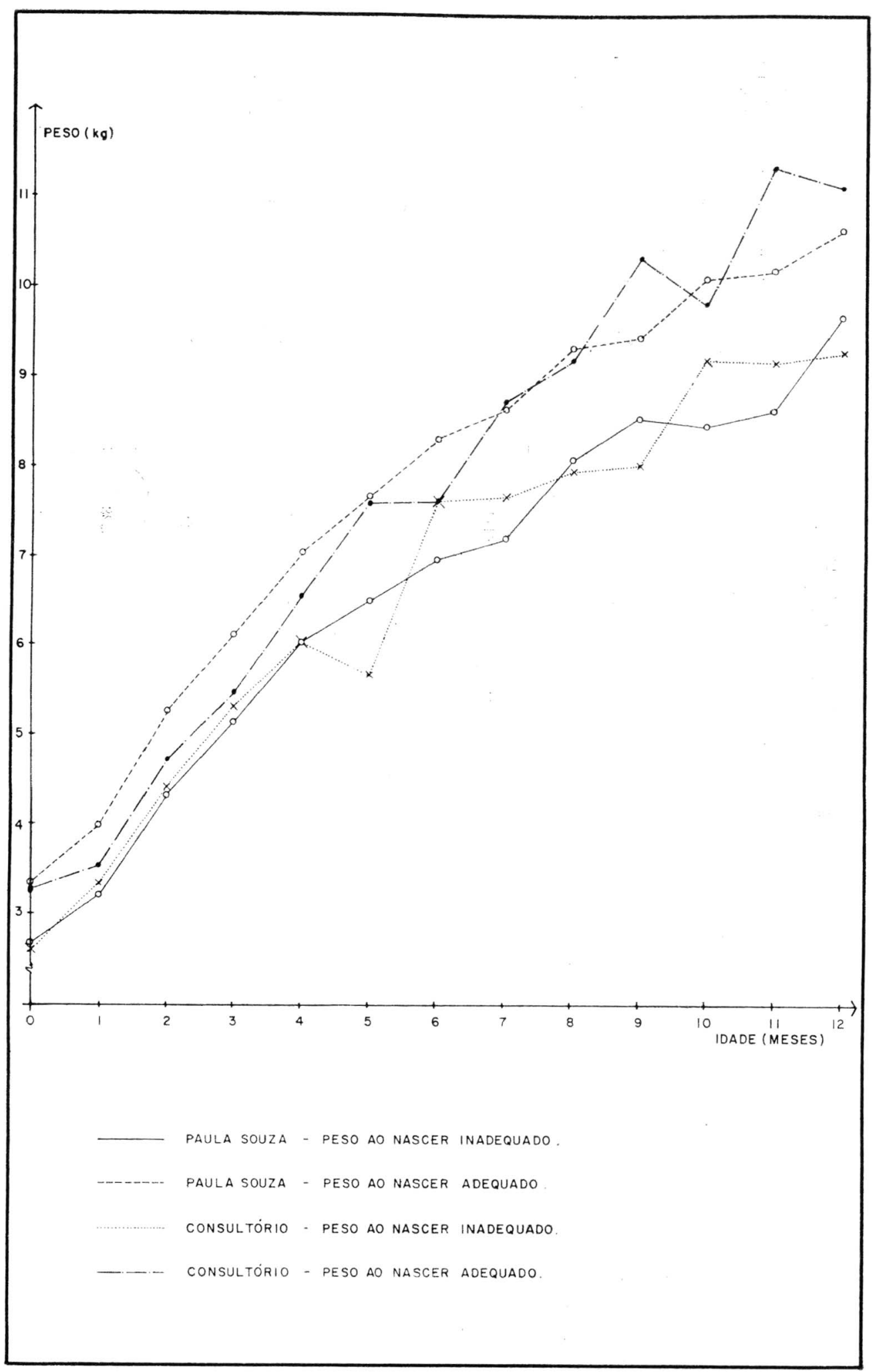

Fig. 1 - Peso ao nascer e mês a mês de crianças com indice ponderal baixo (2,50 e menos). Grupos Paula Souza e Consultório. 


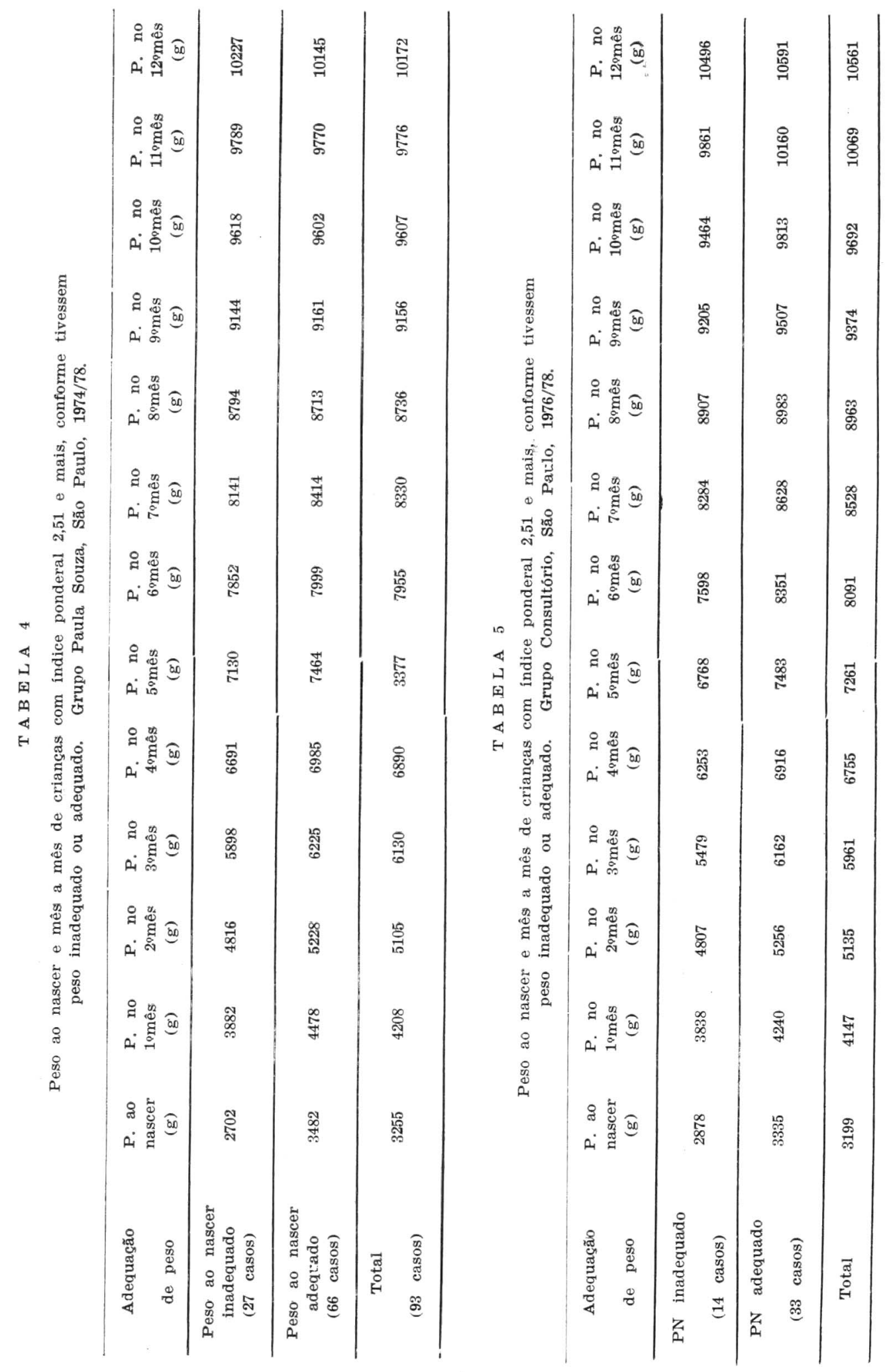




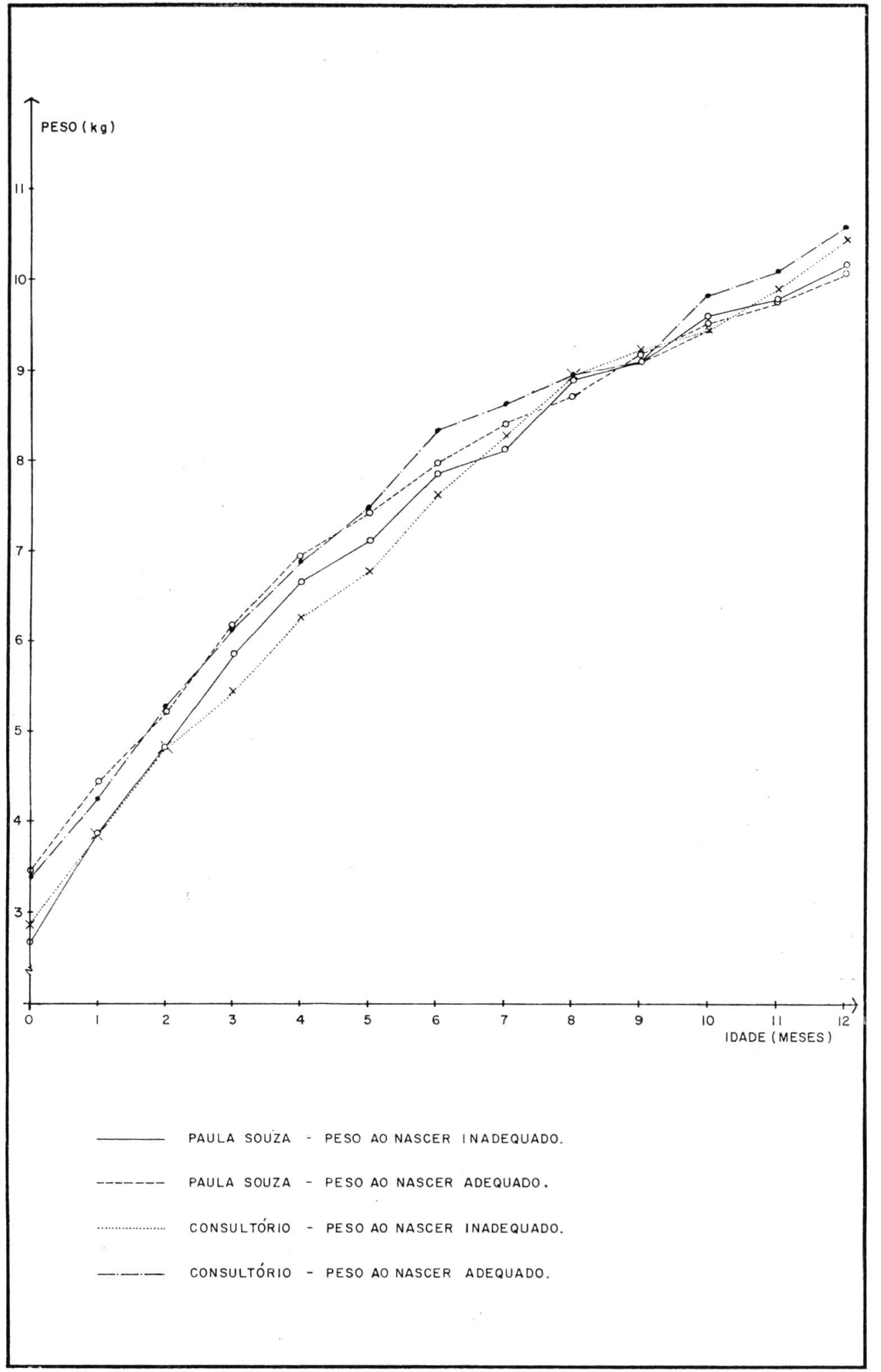

Fig. 2 - Peso ao nascer e mês a mês de criancas com indice ponderal normal (2,51 e mais). riminos Douto Snume n Nonmolthuin 
SIQUeira, A. A. F. de et al. Peso ao nascer, indice ponderal de Röhrer e crescimento pós-natal. Rev. Satte públ., S. Paulo, 14:333-42, 1980.

efeito sinérgico, ao nascimento, entre peso inadequado $e$ indice ponderal baixo, com um prejuizo evidente no ritmo de crescimento posterior.

As Tabelas 4 e 5 e Fig. 2 apresentam muitas semelhanças. Assim é que os valores médios de peso (no total de casos), para cada grupo, são muito semelhantes. As diferenças, mês a mês, poucas vezes ultrapassaram $200 \mathrm{~g}$ (somente no $11^{\circ} \mathrm{e}$ $12^{\circ}$ meses). Os pesos de todos os subgrupos, a partir do $6^{0}$ mês, são muito bons, comparados aos padrōes de crescimento de crianças. $\mathrm{O}$ mais interessante é que, nos dois grupos, as diferenças, para menos, que havia nos primeiros meses, nos subgrupos peso ao nascer inadequado, praticamente desapareceram entre o $6^{\circ}$ e $8^{\circ}$ meses de vida, e não há mais diferença entre os 2 subgrupos, nos 2 grupos, no fim do primeiro ano de vida.

Em outras palavras, crianças que nascem com peso inadequado, mas com indice ponderal normal (ou seja: 2,51 e mais), apresentam um ritmo de crescimento pós-natal acelerado, de tal sorte que após 6 ou 8 meses alcançam, em peso, crianças que nasceram com peso superior a $3.000 \mathrm{~g}$. É como se $O$ indice ponderal normal garantisse um ritmo de crescimento acelerado suficiente mesmo para que crianças nascidas com peso inadequado atingissem pesos altamente satisfatórios logo aos 6 ou 8 meses de idade.

Conclui-se assim que, crianças que ao nascer têm peso igual ou menor que 3.000 g. e indice ponderal igual ou menor que 2,50 correm o risco de apresentar um crescimento (medido por peso) insatisfatório, o que não ocorre quando têm indice ponderal normal.

Interessante é lembrar, além disso, que o cálculo do indice ponderal (e sua associação com o próprio peso do recém-nascido) exige apenas que se conheçam o peso e o comprimento ao nascer, dispensando, assim, a necessidade de conhecer a duração da gravidez, ou seja, a idade gestacional

Talvez a principal dificuldade de estimar o ritmo de crescimento intra-uterino seja o cálculo da idade gestacional, pois nenhum critério clínico (pediátrico ou obstétrico) ou técnica propedêutica, como ultrassonografia ou exame do líquido amniótico, é capaz de determiná-la com precisão; talvez, ainda, a melhor maneira de fazê-lo baseiese na data do inicio da última menstruação, com uma margem de erro de 2 semanas.

Quando se utilizam as curvas de crescimento intra-uterino, verifica-se que, para pesos próximos aos limites inferiores das curvas, uma diferença de uma semana a mais ou a menos na idade gestacional pode mudar a classificação do recém-nascido de pequeno para adequado à idade gestacional e vice-versa.

Esta nova técnica de prognosticar o ritmo de crescimento da criança, não necessitando da idade gestacional, parece ser promissora, ainda mais tendo mostrado comportamento muito semelhante em duas populações diferentes a que foi aplicada. Resta saber se, aplicada a outras populações, continuará mostrando o mesmo comportamento, bem como saber em quais situações nascem crianças de peso inadequado e indice ponderal baixo. 
SIQUEIRA, A. A. F, de et al. Peso ao nascer, fndice ponderal de Röhrer e crescimento pós-natal. Rev. Saúde puibl., S. Paulo, 14:333-42, 1980.

RSPUB9/513

SiqueIRA, A. A. F. de et al. /Birth weight, Rohrer's ponderal index and postnatal birth.] Rev. Saúde públ., S. Paulo, 14:333-42, 1980.

ABSTRACT: The relationship of birth weight, Rohrer's ponderal index and child growth during the first year of life were studied. It is discovered that children born with a low ponderal index of 2.50 or less and a 3.000 gram weight or less suffered growth impairment. At the age of twelve months, they weighed about 1,500 grams less tham those born with a normal ponderal index (2.51 or more). All the children who were born within the normal ponderal index presented approximately the same weight at the end of their first years of life, irrespective of their birth weight. The great advantage of the technique employed is that the period of pregnancy need not be calculated.

UNITERMS: Birth weight. Growth. Infant, low birth weight.

\section{REFERENCIAS BIBLIOGRAFICAS}

1. BECK, G. T. \& BERG, B. J. The relationship of the rate of intrauterine growth of low-birth-weight infants to later growth. J. Pediat., 86:504-11, 1975.

2. BIRCH, H. G. \& GUSSOW, J. D. Disadvantaged children-health, nutrition and school failure. New York, Grunne-Stratton, 1970 .

3. CHASE, H, C. International comparisons of perinatal and infant mortality. Vital Hith Statist. Ser., 3(6), 1967.

4. CHOW, B. F. et al. Maternal nutrition and metabolism of the offspring: studies in rats and men. Amer, $J$. publ. Hlth, 58:668-77, 1968.

5. DOBBING, J. The later growth of the brain and its vulnerability. Pediatrics, $53: 2-6,1974$.

6. GRUENWALD, P. Growth of the human fetus. I. Normal growth and its variation. Amer. J. Obstet. Gynec., $94: 1112-9,1966$.

7. HSUEH, A. M. et al. The importance of the period of dietary restriction of the dam on behavior and growth in the rat. J. Nutr., 104:37-46, 1974.

8. LINDELL, A. Prolonged pregnancy. Acta obstet, gynec. scand., 35:136-63, 1956.
9. LUBCHENCO, $L$. $O$. et al. Intrauterine growth as estimated from l.ve-burn birth-weight data at 24 to 42 weeks of gestation. Pediatrics, $32: 793-800$, 1963.

10. MARCONDES, E. et al. Estudo antropométrico de crianças brasileiras le zero a doze anos de idade. An. Nestle, (84) :1-200, 1970.

11. METCOFF, J. et al. Biomolec::lar studies of fetal malnutrition in maternal leucocytes. Pediatrics, 47:180-91, 1971.

12. MILLER, H. C. \& HASSANEIN, $\mathbf{K}$. Diagnosis of impaired fetal growth in newborn infants. Pediatrics, 48:511 22, 1971.

13. MILLER, H, C. \& HASSANEIN, K. Fetal malnutrition in white newborn infan:s: maternal factors. Pediatrics, 52:504-12, 1973.

14. MILLER, H. C. \& HASSANEIN, K. Maternal factors in "fetally malnourished" black newborn infants. Amcr. $J$. Obstet. Gynec., 118:62-7, 1974.

15. MONTEIRO. C. A. O peso ao nascer no municipio de São Paulo: impacto sobre os niveis de mortalidade na infancia. São Paulo, 1979. [Tese de Dorltoramento - Faculdade de Saúde Pública da USP]. 
SIQUEIRA, A. A. F. de et al. Peso ao nascer, Indice ponderal de Röhrer e crescimento pós-natal Rev. Saúde públ., S. Paulo, 14:333-42, 1980.

15. NISWANDER, K. \& JACKON, E. C. Physical characteristics of the gravida and their association with birth weight and perinatal death. Amer. J. Obstet. Gynec., 119:306-13, 1974.

17. PUFFER, R. R. \& SERRANO, C. V. Patterns of mortality in childhood. Washington, D. C., Pan American Health Organization, 1973. (PAHOScient. publ., 262).

18. SIQUEIRA, A. A. F. de Mortalidade neonatal e prematuridade. São Paulo, 1974. [Dissertação de Mestrado - Faculdade de Saúde Pública da USP].

19. TANNER, J. M. \& THOMSON, A. M. Standards for birth weight at gestation periods from 32 to 42 weeks. allowing for maternal heigth. Arch. Dis. Child., 45:566-9, 1970.

20. USHER, R. \& MCLEAN, F. Intrauterine growth of live-born causasian infants at sea level: 7 dimensions of infants born between 25 and 44 weeks of gestation. J. Pediat., 74:901-10, 1969.

21. WINICK, M. Malnutrition and brain development. New York, Oxford University Press, 1976.

22. YERUSHALMY, J, et aI, Birth weight and gestation as indices of "Immaturity". Amer, J. Dis. Child., 109:43-57, 1965

Recebido para publicasāo em 19/0s/1980

Aprovado para publicacão em 23/06/1980 